\title{
Electroanalysis of nirofurazone at silver particles and graphite powder composite electrode
}

\author{
J. Zoubir ${ }^{1} \cdot$ A. Assabbane ${ }^{1} \cdot$ I. Bakas ${ }^{1}$
}

Received: 13 September 2021 / Revised: 3 November 2021 / Accepted: 11 November 2021 / Published online: 2 December 2021

(c) Korean Carbon Society 2021

\begin{abstract}
The main objective of the research was to deposit thin films of silver on a graphite carbon paste in a phosphate buffer medium using an electrochemical method. To construct a nitrofurazone detection sensor that is highly sensitive. Different manufacturing parameters, such as electrodeposition potential, $\mathrm{pH}$ effect, potential scan rate effect, and number of scan cycles, were examined in this section. The parameters were optimized to improve the deposited silver layers various electrocatalytic characteristics. The Nitrofurazone reduction process is diffusion controlled, as seen by the linear variation of Epc with $\log (\mathrm{v})$. The constructed Ag-NPs@CPE electrod has excellent electrical characteristics a large active surface area and low background with extremely high electrical conductivity, according to structural and electrochemical characterizations such as Scanning electron microscopy, X-ray diffraction (XRD) and cyclic voltammetry. The constructed sensor has a very remarkable analytical performance for nitrofurazone molecule identification, with a very low detection limit of about $10^{-8} \mathrm{M}$. The detection of nitrofurazone using our Ag-NPs@CPE sensors in real samples contaminated with the antibiotic nitrofurazone, such as tap water and urine. In the selected sample, the electroanalytical findings reveal a very satisfactory recovery rate of more than 94 percent.
\end{abstract}

Keywords Nitrofurazone $\cdot$ Differential pulse voltammetry·Ag-NPs@CPE sensor · Electrocatalysis

\section{Introduction}

Antibiotic contamination is a major global issue due to the negative effects of antibiotic usage and mismanagement on ecosystems and human health. Antibiotic residue detection in food is important, but scientists find it difficult to analyze accurately and efficiently. In this sense, the European Community decided in 1993 to prohibit the use of different nitrofurans (furaltadone, nitrofurantoin, and nitrofurazone) in food animals to protect human health $[1,2]$. Nitrofurazone (5-nitro-2-furaldehyde semicarbazone) is a nitrofuran derivative exhibiting antibacterial action against a diversity of gram-negative and gram-positive bacteria [3, 4]. It is also an anti-infective used to treat or monitor infections of the skin, genitourinary tract, eye, nose, and ear [5, 6]. The vast majority of medicines are The pharmacokinetic half-life of Nitrofurazone (NFZ) tablets or injections is quite short.

\section{J. Zoubir}

zoubirjallal@yahoo.fr

1 Team of Catalysis and Environment Ibn Zohr University Faculty of Sciences, BP 8106 Agadir, Morocco
The excretion is renal, with $40 \%$ of it in an active condition, which explains the activity of the urinary tract. Oral absorption is quite high. Nitrofurans are found in breast milk [7]. In veterinary medicine it's used to treat and prevent coccidiosis in chickens and cattle as well as to treat necrosis. Nitrofurazone is a potential teratogen and carcinogen that enters the human body via the food chain and is consumed through food and food products. The year was 1995. The Chinese Ministry of Agriculture has declared that the use of nitrofurazone for humans and animals is prohibited [8]. As a consequence, to provide better protection against contamination issues, it is essential in developing a method of analysis that is sensible, rapid, workable and effective for analyzing the active principle of Nitrofurazone in real-world samples.

Several methods have been developed and applied to detect traces of Nitrofurazone active substances, including high performance liquid chromatography (HPLC) [9], Luminescence [10] and liquid chromatography tandem mass spectrometry [11] These techniques are applied to the detection of Nitrofurazone in a variety of real samples, but they are often time and/or cost intensive and expensive to implement and apply in laboratory diagnostic practice. The preparation 
of modified electrodes for detection through numerous intermediates that significantly and rapidly modify the behavior of drug molecules to facilitate their detection is the most important subject in this context of electroanalytical methods oriented toward the analysis of drug molecules. One of the most established research subject in electrochemistry since 1979 has been the chemical modification of carbon paste electrodes[12]. So far, several forms of carbon-based electrodes have emerged in recent years for detection of target pharmaceutical substances in biological fluids and different forms of assay Nagaraj P. Shetti group [13, 14]. The synergistic impact of carbon-based materials with a combination of various modifiers such as metal nanoparticles [15], metal oxides [16-18], conductive polymers or surfactants $[13,19]$, enhances the competence of the electrode in the electrochemical determination of bioactive and electroactive molecules. The utilization of carbon-based materials in the development of sensing electrodes is still in its infancy. Thus, future research should be directed towards a better understanding of the structural properties of carbon-based materials that manage the kinetics of electron transfer to the electrode surface and avoid the formation of modifier precipitation [20,21]. Among the best known research groups in this field are Tejraj M. Aminabhavi, Nagaraj P. Shetti and all of them have developed several electrochemical sensors for the detection of pharmaceutical substances in different real samples with very low detection limits. The novelty of our work has been directed towards this axis to develop an innovative strategy to build new materials with particularly interesting properties. We have combined carbon graphite nanomaterials and metallic silver nanoparticles to build new electrochemical sensing devices by simple and efficient electrodeposition processes that will open new fields of applications in nanoscience and nanotechnology. Silver nanoparticles have received much attention in the last two decades, especially in the fields of electrocatalysis [18] due to their large electrochemical window, excellent electrical conductivity, chemical and thermal stability, and intrinsic electrocatalytic activity. The electrochemical techniques have also shown the advantages of a simple less time-consuming extremely cost-effective and environmentally friendly pretreatment strategy [13]. Due to their low cost, high sensitivity and superior selectivity [21]. In fact, the presence of reducible $\mathrm{NO}_{2}$ groups in the side chain of Nitrofurazone facilitates its detection on the electrocatalytic surface of modified working electrodes with current electrochemical techniques. In this approach. The objective of our research is to develop a thin silver deposit on a carbon paste to create a highly sensitive sensor to Nitrofurazone molecules with excellent electrochemical performance and that the interaction between Nitrofurazone molecules and functional silver metal nanoparticles present on the surface of the constructed electrode is properly explored.
In this study, silver particles were used that were electrodeposited on carbon paste electrodes. Using cyclic voltammetry, this technique is ideally suited for the synthesis of metallurgical nanostructures and pure thin films of excellent quality, shape, and well-controlled surface states. Electrocatalytic reduction and Nitrofurazone determination studies have been investigated using cyclic voltammetry, chronoamperometry, and differential pulse voltammetry. The experimental parameters that effect the shift and intensity of the peak potential were evaluated and the catalytic parameters of the electrode process were discussed in detail. Finally, using the proposed method this electrode was successfully used to determine Nitrofurazone traces in real samples.

\section{Experimental section}

\subsection{Chemicals and reagents}

All of the chemical products used in this study were of analytical quality or were of the highest purity available. Or the purest form of the available pure. Potassium nitrate, sodium hydroxyde, silver nitrate, acetic acid, phosphoric acid, and boric acid were obtained from Merck (Darmstadt, Germany), Fluka (St. Gallen, Switzerland), and Riedel de Haen (Seelze, Germany) and used accordingly. To prepare 1.0 $10^{-3}$ mol. $\mathrm{L}^{-1}$ of Nitrofurazone mother solution, the Nitrofurazone purchased from Sigma-Aldrich was dissouted in $0.1 \mathrm{~mol} \mathrm{~L}^{-1}$ of tampon phosphate buffer (PBS; pH 7) The solutions étalons de travail have been prepared by diluting the mother solutions. Paraffine was used as a bonding liquid for the carbon paste electrode unmodified (CPE). The carbon paste was supplied from Carbone Lorraine (Lorraine France ref. 9900). All these experiments were performed with distilled water and at room temperature.

\subsection{Apparatus}

With the help of an Origa Master 5 potentiostat connected to an electrochemical cell, an électro-catalytic deposit of metal was achieved. The electrochemical cell is made up of an silver nitrate solution $\left(\mathrm{Ag}^{+}+\mathrm{NO}_{3}{ }^{-}\right)$and three electrodes one of which is a graphite carbon paste electrode that serves as a work electrode for the electrodeposition. A reference electrode (RE) $\mathrm{Ag} / \mathrm{AgCl}$ saturated with $0.3 \% \mathrm{KCl}$ and a platine wire as a counter electrode to provide current (AE). All experimental dates and results were entered onto a personal computer that was connected to an Origa Master 5 potentiostat. Using the same potentiostat the Origa Master 5 , measurements of spectroscopy of electrochemical impedance and cyclic voltammetry were also carried out. The diffraction of X-rays of type PERT-PRO (BRUKER-AXS) with the radiation $\mathrm{Cu}_{\mathrm{K}}=1.5406$ installed at the University $\mathrm{Ibn}$ 
Zohr of Agadir in Morocco was used to determine the nature of various chemical compositions in a silver deposit. The image of the distribution of silver nanoparticles on graphite carbon sheets was seen using a JEOL scanning electron microscope installed at the research unit of Ibn Zohr University in Agadir, Morocco. Using high quality $\mathrm{pH}$ meters several measurements of $\mathrm{pH}$ values were performed.

\subsection{Process for modifying CPE}

The unmodified (CPE) were created using the following procedure [22]. A portion of the homogeneous paste was carefully positioned in the electrode cavity (fabricated in the lab, with a geometrie of $0.1256 \mathrm{~cm}^{2}$ ) and polied with a smooth piece of paper. To make the electrical connection, a carbon-based bar was used. The unmodified carbon paste (CPE) was immersed in an electrochemical cell containing $0.5 \mathrm{mmol.L}-1$ of $\mathrm{AgNO}_{3}$ diluted in $0.1 \mathrm{M}$ of phosphate buffer $(\mathrm{pH}=2)$ electrolyte solution to deposit Ag particles. Using cyclic voltammetry, the Ag particles were fixed on the electrode surface for 2 cycles between potential ranges of 00 to $800 \mathrm{mV}$. (8 segments). After that, the electrode was placed in a cell with $0.1 \mathrm{M}$ of phosphate buffer $(\mathrm{pH}=2)$ and Nitrofurazone. In the potential range of 00 to $-1200 \mathrm{mV}$ cyclic voltammetry and differential pulse voltammetry were used. All experiments were carried out successfully and in a systematic manner consistent with current standards.

\subsection{Electrochemical measurement}

All electrochemical experiments were performed with an electrode produced by silver electrodeposition under optimal conditions that were met in all electrochemical experiments performed in this study. The cyclic voltammetric method was used to perform electrochemical deposition of silver on carbon graphite. The cyclic voltammograms of Nitrofurazone detections were recorded in the potential range from $00 \mathrm{mV}$ to $-1200 \mathrm{mV}$. In addition, the recovery ratio was calculated using the calibration curve with regression of the equation. This was traced using differential pulse voltammetry. All electrochemical experiments were carried out with the electrode constructed via silver electrodeposition under the optimum conditions listed in Table 1.

\subsection{Analytical procedure}

To avoid contamination with Covid 19 viruses, human urine samples were obtained from a local clinic near Ibn Zohr University in Agadir Morocco, and tap water samples were obtained from the laboratory. The urine samples were centrifuged at $3000 \mathrm{tr} / \mathrm{min}$ for $15 \mathrm{~min}$ (sigma mode Laborzentrifugen TM10155), then smoothed in a refrigerator set at $10{ }^{\circ} \mathrm{C}$ for $4 \mathrm{~h}$ to facilitate phase separation, before being filtered
Table 1 Different parameters optimized during the silver electrodeposition process

\begin{tabular}{ll}
\hline Influence of parameter variables & Optimizes \\
\hline The supporting electrolyte & Phosphate buffer (PBS) \\
The sweep rate of the potential & $10 \mathrm{mV} / \mathrm{s}$ \\
$\mathrm{pH}$ & 2 \\
The concentration of silver ions $\mathrm{Ag}^{+}$ & $0.5 \mathrm{mmol} / \mathrm{L}$ \\
The number of scan cycles & 2 \\
\hline
\end{tabular}

through $0.85 \mu \mathrm{m}$ pore diameter qualitative filter paper. During the analysis the remaining real samples were not pretreated. To obtain concentrations of $10^{-5} \mathrm{M}, 510^{-6} \mathrm{M}$ and $10^{-6}$ M. $5 \mathrm{~mL}$ of urine filtrates were measured and transferred into a $100 \mathrm{~mL}$ container containing phosphate buffer at $\mathrm{pH}=8$ and spiked with a quantity of the Nitrofurazone. We used the same technique for the other actual tap water samples as we used for the real urine samples to obtain concentrations in the range of $10^{-5} \mathrm{M}, 510^{-6} \mathrm{M}$ and $10^{-6} \mathrm{M}$ of Nitrofurazone. All of the contamination processes for the real samples were carried out in our laboratory in the same way, under the same working conditions, and within the same analysis time period. The differential pulse voltammetry were registered under the evaluated optimal conditions and then analyzed to obtain more precise results.

\section{Results and discussion}

\subsection{Electrochemical behavior of the Nitrofurazone}

The results of individual $\mathrm{CV}$ tests in a tampon phosphate buffer $(\mathrm{pH}=7)$ solution containing $1.010^{-3} \mathrm{M}$ of Nitrofurazone are shown in Fig. 1 using unmodified carbon paste (CPE and (Ag-NPs@CPE), respectively. In the potential range between 00 and $-1200 \mathrm{mV}$, both electrodes showed a reduction response towards Nitrofurazone. Comparing the behavior of the unmodified (CPE) and (Ag-NPs@CPE) electrodes the cathodic potential of the Nitrofurazone was positively shifted from $-680 \mathrm{mV}$ to $-580 \mathrm{mV}$ when the modified electrode was used. In addition to the $100 \mathrm{mV}$ potential shift, the current of pic reduction has increased at the modified Ag-NPs@CPE electrode in comparison to the unmodified carbon paste (CPE). This demonstrates the rapidity with which electrons are transferred to the surface of the modified electrode. Furthermore, no signals of oxidation were observed in the direction opposite to the anodic scan in the volammograms of both electrodes. This shows that the behavior of Nitrofurazone is irreversible at the modified electrodes constructed by electrodeposition.

As a result, the displacement of the peak towards positive potentials and the growth of the current of the Nitrofurazone 
Fig. $1 \mathrm{CVs}$ of Nitrofurazone on the electrode constructed of AgNPs@CPE and unmodified CPE in PBS $(\mathrm{pH}=7)$ with a scan rate of $20 \mathrm{mV} / \mathrm{s}$

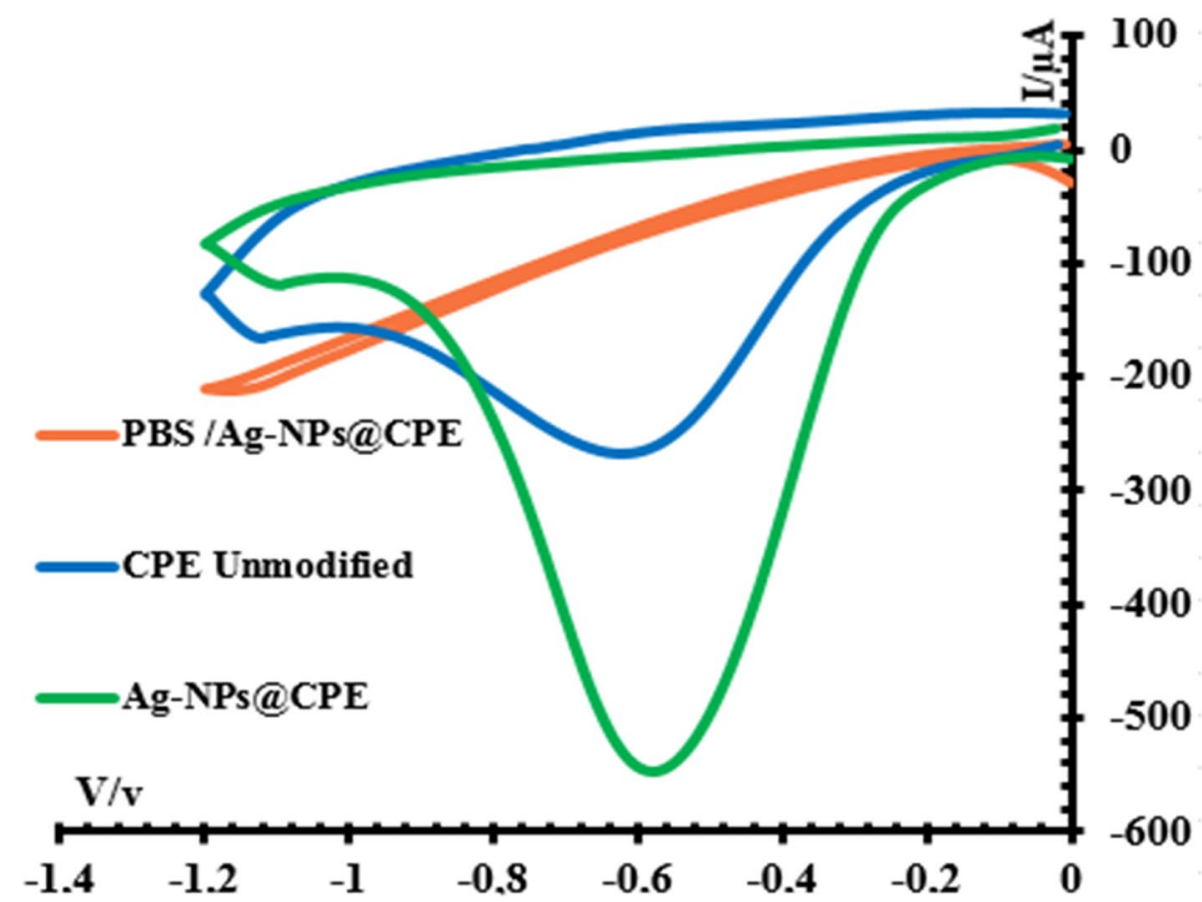

reduction peak on the surface of the proposed electrode are attributed to the presence of a group of silver atoms of nanometric size which increase the electrocatalytic power of the Nitrofurazone reduction reaction and allow a significant increase in the number of molecules participating in the Nitrofurazone reduction reaction on the surface of the electrode constructed by silver nanoparticles which facilitate us the conversation of the $\mathrm{NO}_{2}$ attractor group of Nitrofurazone molecules into $\mathrm{NHOH}$ group in an adequate and efficient way.

\subsection{Electrodeposition of Ag on Carbon Graphite}

The voltammograms obtained during the electrodeposition of silver nanoparticles on the surface of a graphite paste were measured using a cyclic voltammetry in the potential range of $800 \mathrm{mV}$ to $00 \mathrm{mV}$ using an silver-containing phosphate buffer (Fig. 2). The obtained results show that the chosen potential range is very beneficial to reducing ions of silver. Furthermore, the ion reduction peak is found at $0.43 \mathrm{~V}$ versus $\mathrm{Ag} / \mathrm{AgCl}$, indicating that the silver electrochemical behavior is completely reversible, as evidenced by the cyclic voltammograms. When the number of cycles increases, the intensity of the redox current decreases indicating that the nucleation of argent atomes is halted due to the saturation of the electrode surface which pushes silver ions into solution while blocking electron transfer to the surface. As a result of this blockage the reduction of $\mathrm{Ag}^{+}$ions on the surface of the electrode has become difficult limiting the reduction reaction of ions $\mathrm{Ag}^{+}$.
When employed in semi-continuous or continuous mode, the proposed electrochemical sensor must support a large number of electrochemical measurements as well as the number of regeneration steps of the agent layer placed on the carbon paste to continue the electro-catalytic characteristics. We ve started optimizing all of the electrodeposition process experimental parameters such as the $\mathrm{pH}$ of the electrolyte solution, the balayage speed, the number of cycles, and the silver concentration in the electrolyte solution. This method allows us to improve the electro-catalytic performance of the silver-based Nitrofurazone detection layer.

\subsection{Optimization of Ag film deposition parameters on carbon graphite}

To obtain more homogeneous Ag-NPs deposits and a better reproducibility, it is necessary to control the electrodeposition process. To this end, we have optimized the deposition parameters of the Ag film on graphite carbon, several electrolysis times have been tested, then a follow-up of the functionalization has been performed in a regular and systematic way to improve the quality of the Ag-NPs deposits and to guarantee a better electrocatalytic activity. All operating parameters are presented in Table 1.

The influence of the $\mathrm{pH}$ of the support electrolyte on the silver electrodeposition was the first parameter investigated (Fig. 3A). The $\mathrm{pH}$ range was 1 to 5 . In the phosphate buffer electrolyte solution with $\mathrm{pH}=2$ the best results (in terms of current intensity and displacement of the Nitrofurazone cathodic peak) were attained. This suggests that 


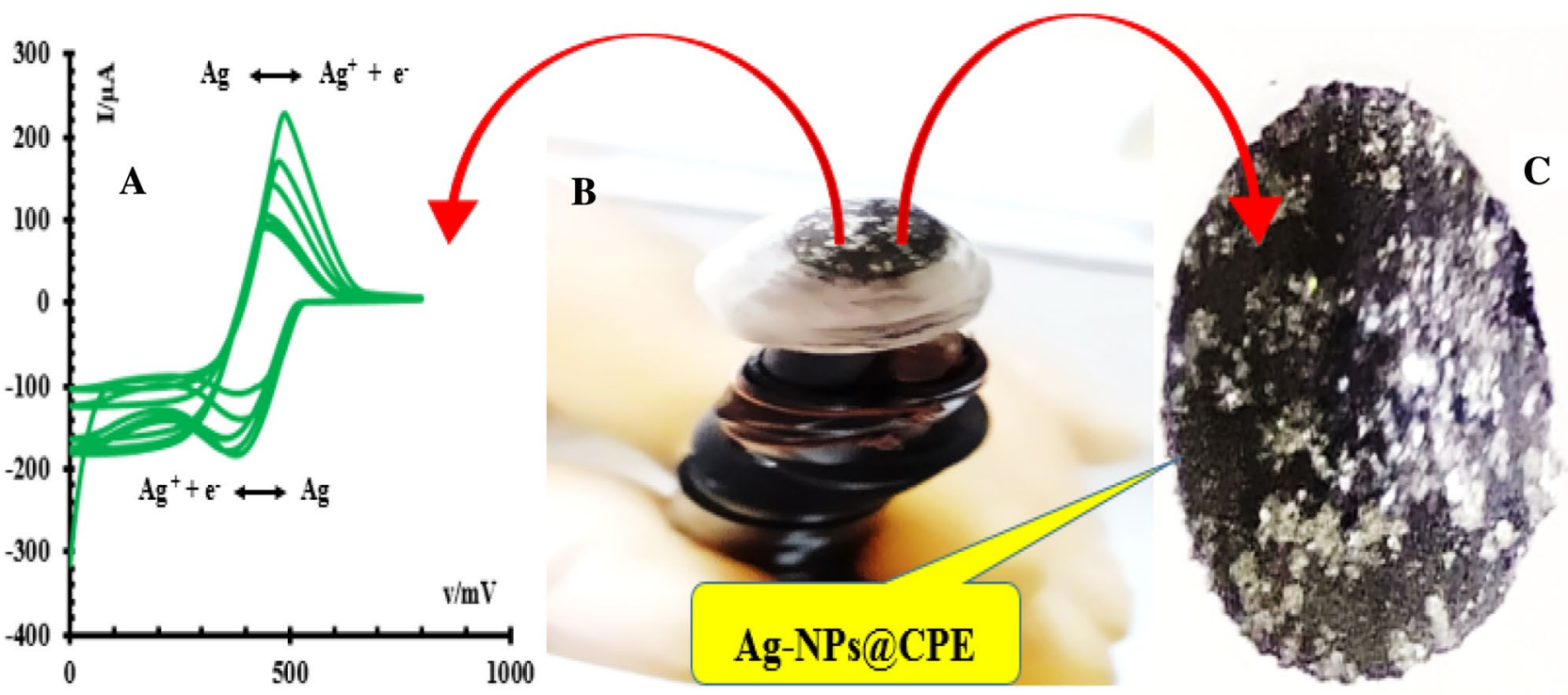

Fig. 2 CVs of silver electrodeposition on graphite carbon paste (A). The real image of the working electrode with electrochemical Ag deposition (B). The image of the carbon paste constructed with silver deposition (C)

at $\mathrm{pH}=2$ the appropriate nucleation of silver microparticles on carbon was achieved, resulting in strong electrocatalytic activity for the reduction of Nitrofurazone molecules. As a result the phosphate buffer at $\mathrm{pH}=2$ is ideal for conducting silver electrodeposition on graphite carbon.

Figure 3A shows the effect of the Ag electrodeposition scan rate on the Nitrofurazone reduction electrocatalytic current intensity. When performing the silver electrodeposition the highest performance of the constructed electrode was obtained with a potential scan speed of $\mathrm{v}=10 \mathrm{mV} / \mathrm{s}$ according to the data. Higher electrodeposition speed on the other hand reduces the sensitivity of our constructed electrode, because the processes of silver microparticle nucleation and creation during electrodeposition are difficult to detect and observe using cyclic voltammetry

The electrodeposition process is influenced by the concentration of reactive Ag ions. As a result, the effect of Ag ion concentration on the Nitrofurazone reduction process was investigated using $\mathrm{AgNO}_{3}$ dissolved in a solution containing $0.1 \mathrm{~mol} \mathrm{~L}^{-1}$ the tompon phosphate solution at concentrations ranging from $1.010^{-4}$ to $1.010^{-3} \mathrm{~mol} \mathrm{~L}^{-1}$ $\mathrm{AgNO}_{3}$ (Fig. 3C). At $0.5 \mathrm{mmol} \mathrm{L}^{-1} \mathrm{AgNO}_{3}$ the optimum response for Nitrofurazone detection was obtained; however, beyond this concentration, a poor reaction was seen. Due to the high silver concentration of the solution this event was attributed for the formation of a silver film on the carbon graphite. Therefore, the optimal concentration for silver electrodeposition is defined around $0.5 \mathrm{mmol} \mathrm{L}^{-1}$ of $\left[\mathrm{AgNO}_{3}\right]$ in the further research and development work done in the laboratory.
The performance of the constructed electrode also depends on the number of scan cycles. According to the results obtained (Fig. 3D). The number of scan cycles that provides excellent electro-catalytic activity to detect Nitrofurazone was about $n=2$ which means that the electrode area is not completely covered with a thin film of silver microparticles generating a strong electron exchange capacity with the attractive groups of Nitrofurazone molecules. Nevertheless, the increase in the number of scanning cycles generates a layer of silver microparticles of very high mass that significantly decreases the sensitivity of the electrode area.

\subsection{Characterization by X-ray diffraction}

The recording of the spectre of diffraction of X-rays confirmed the nucleation of silver atomes on carbon-based paste under optimal conditions. In comparison to the graphite carbon spectre alone the obtained spectrum reveals the presence of several recent pics.

The creation of silver particles can be seen in the X-ray diffraction examination of the silver placed on the carbon paste. Three metallic silver peaks at $2 \theta^{\circ}$ of $38.18^{\circ}, 46.62^{\circ}$, $63.01^{\circ} 66.42^{\circ}$ and $76.4^{\circ}$ witness to the metallic condition of the silver placed on the carbon paste and its uniform distribution across the whole surface of the carbon paste and on the carbon sheets [22, 23] (Fig. 4). Therefore, the creation of a set of silver particles on the surface of the working electrode with a very homogeneous distribution, reflects a large active surface that ensures a greater chance of contact with 

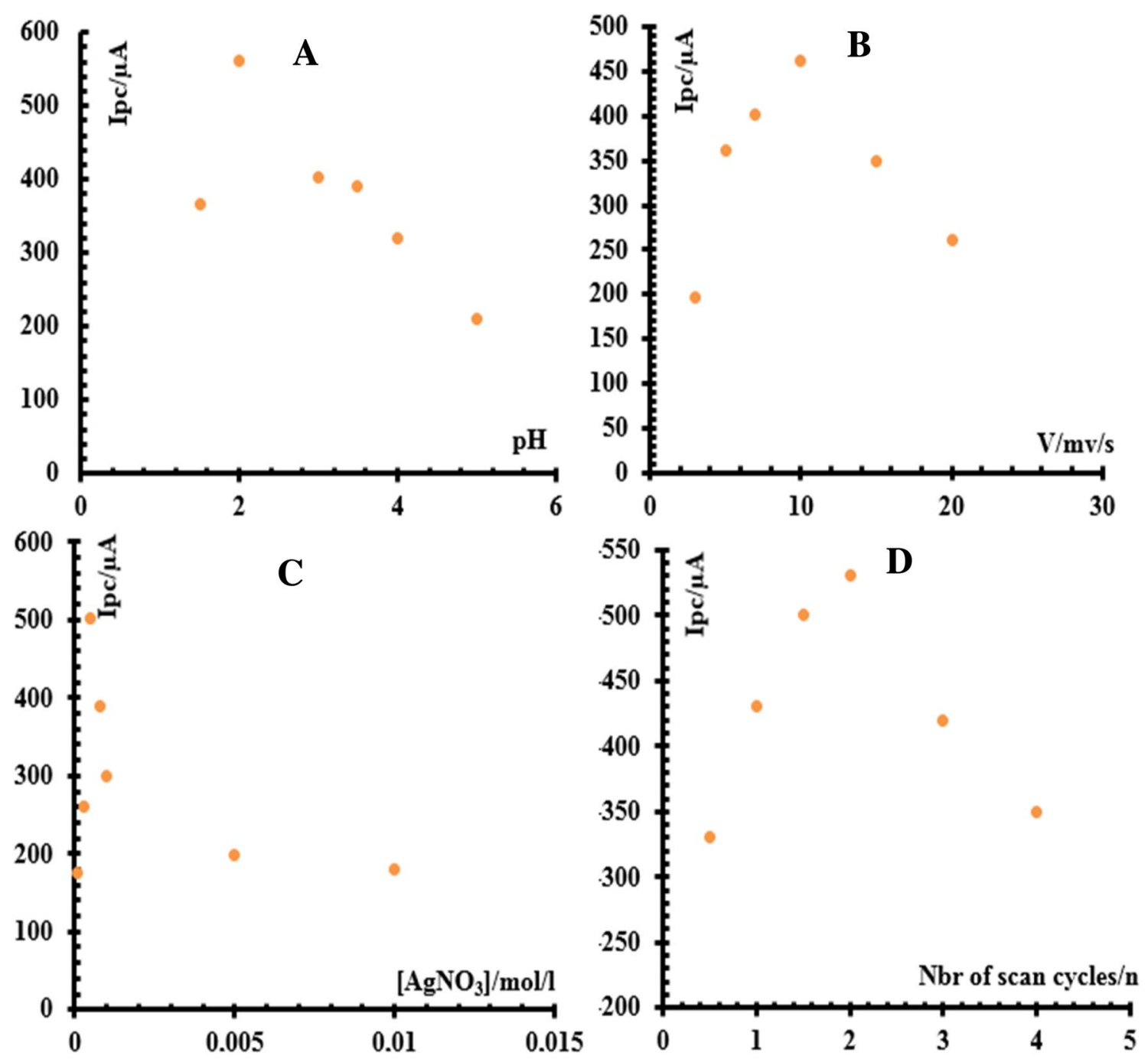

Fig. 3 Curves $\mathbf{A}, \mathbf{B}, \mathbf{C}$ and $\mathbf{D}$ show the influence of experimental parameters of Ag electrodeposition on graphite carbon on the intensity of the reduction peak of $10^{-3} \mathrm{~mol} / \mathrm{L}$ Nitrofurazone in tompon phosphate solution at $\mathrm{pH}=7$

the electroactive substances of the Nitrofurazone, as well as a strong electro-catalytic effect that allows the conversion of the $\mathrm{NO}_{2}$ attraction groups of the side chain of the Nitrofurazone molecules into $\mathrm{NHOH}$ groups.

\subsection{Scanning electron microscopy characterization of Ag-NPs@CPE}

To confirm the silver electrodeposition on the carbon sheets and to analyze the surface of the constructed modified electrodes, we performed scanning electron microscope analyses. The scanning electron microscopy image obtained for the Ag-NPs@CPE electrode is shown in Fig. 5 with EDX analysis.

Figure 5A demonstrates the scanning electron microscopy image of the electrochemical deposition on carbon graphite paste. The paste surface is largely covered with silver nano particles of extremely comparable size that are distributed throughout the paste's entire surface. This result confirms that the nucleation of silver nano particles is immobilized on the majority of the carbon graphite paste surface, and that the Ag atoms produced by the reduction reaction of $\mathrm{Ag}^{+}$ions have been prompted in an Ag nanostructure on the surface of our elaborated electrode, providing a good electro-catalytic capacity. And that the range of potentials used during Ag electro-deposition is ideal for the formation of crystals a silver layer on the carbon graphite sheets [22, 23]

EDX analysis Fig. 5B indicates the existence of silver peaks at $0.4 \mathrm{keV}, 3 \mathrm{keV}, 3.3 \mathrm{keV}$, and $3.5 \mathrm{keV}$. As well as a carbon peak. All of these accurate quote to the presence of significant silver atomic nuclei. The results obtained show good coherence with the information furnished by the X-ray diffraction discussed in the preceding section. 


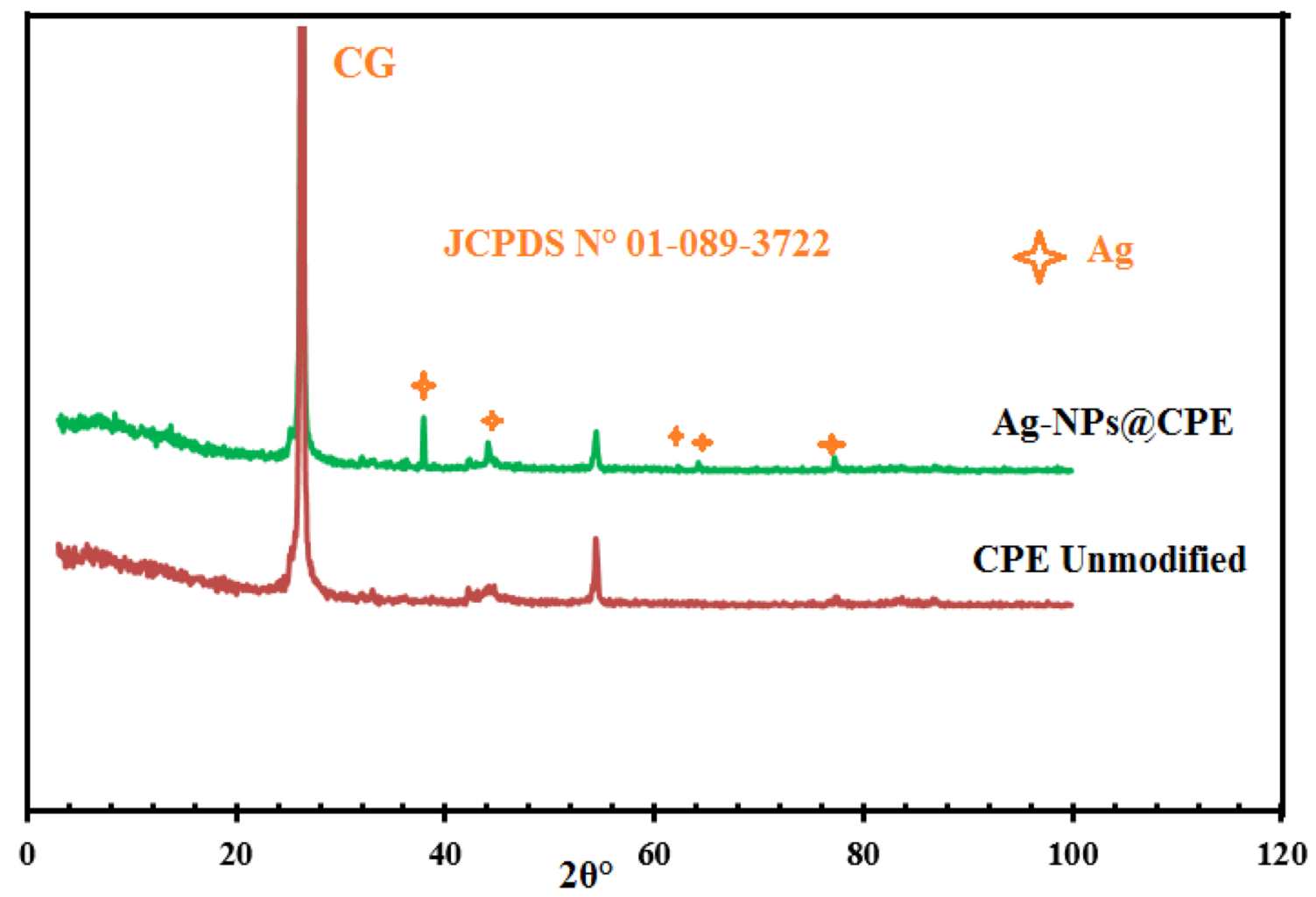

Fig. 4 X-ray diffraction (XRD) of unmodified carbon paste (CPE) and Ag-NPs@CPE constructed
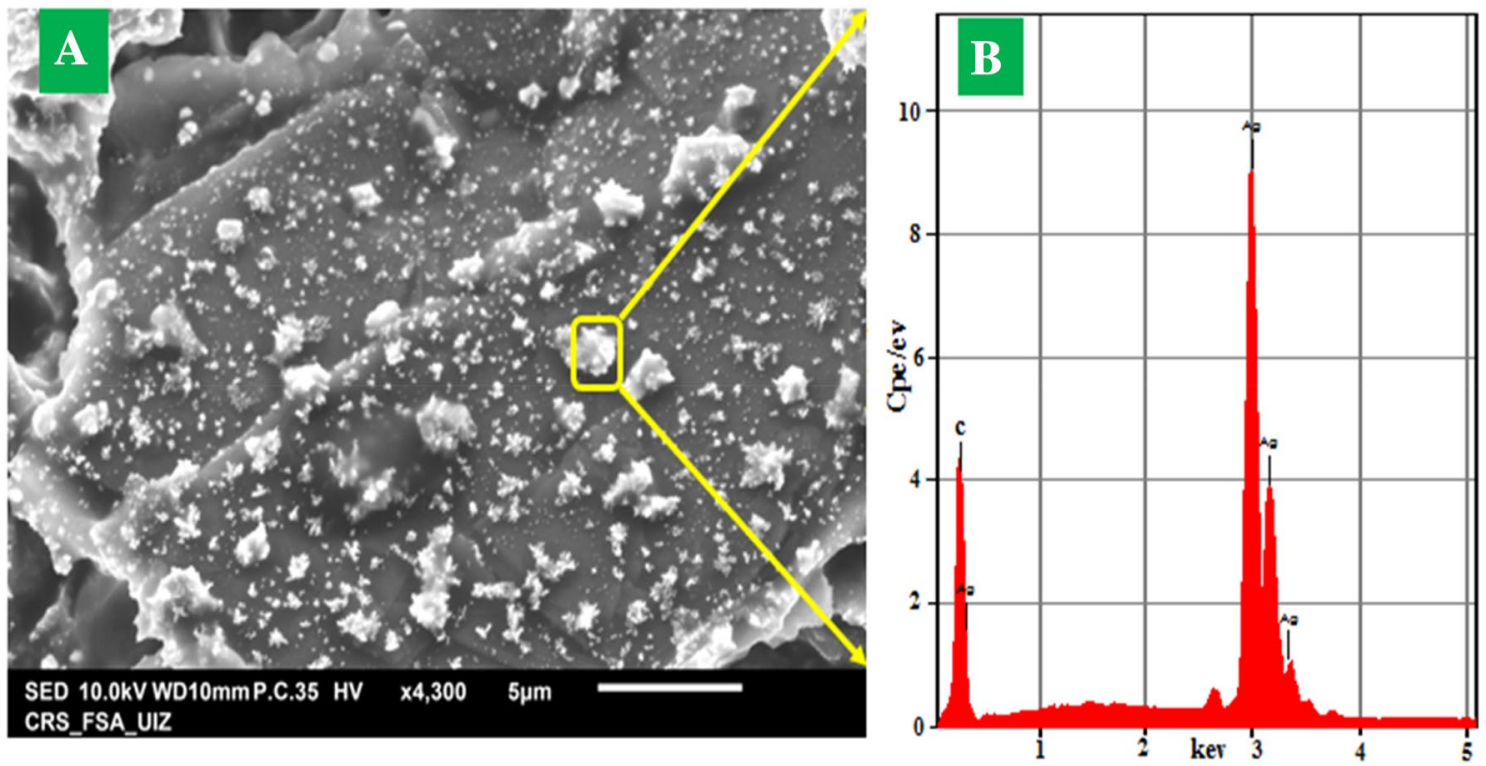

Fig. 5 SEM image of Ag-NPs@CPE constructed (A), EDX spectra (B) 


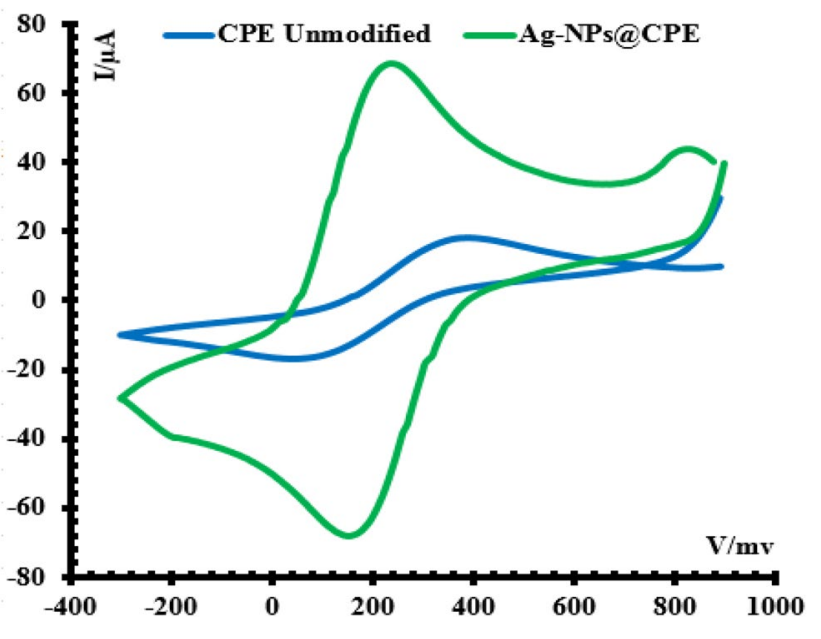

Fig. 6 CVs of unmodified CPE and constructed Ag-NPs@CPE registered in $\mathrm{Fe}(\mathrm{CN})_{6}{ }^{3-/ 4-} 1 \mathrm{mM}$ containing $0.1 \mathrm{M} \mathrm{KCl}$ with at $50 \mathrm{mV} . \mathrm{s}^{-1}$

\subsection{Characterization by cyclic voltammetry}

On the unmodified CPE electrode and the Ag-NPs@CPE electrode constructed with $1 \mathrm{~m} \mathrm{M} \mathrm{Fe}(\mathrm{CN})_{6}^{-3 / 4}$ and $0.5 \mathrm{M} \mathrm{KCl}$ the electrochemical behavior of $\mathrm{M} \mathrm{Fe}(\mathrm{CN})_{6}^{-3 / 4}$ was investigated (Fig. 6). When the current intensity of the oxidation and reduction peaks of the $\mathrm{Fe}(\mathrm{CN})_{6}{ }^{-3 / 4}$ redox probe on the electrode constructed by Ag-NPs@CPE silver electrodeposition is compared to the unmodified electrode (CPE), the current intensity of the oxidation and reduction peaks of the $\mathrm{Fe}(\mathrm{CN})_{6}^{-3 / 4-}$ redox probe on the electrode constructed by Ag-NPs@CPE silver electrodeposition increases almost 5 times. This augmentation highlights the significance of metallic Ag nanoparticle matrices electrochemically synthesized on highly conductive graphite carbon sheets in facilitating the ferrocene ion reduction process at the Ag-NPs@CPE electrode's surface. The potential difference $\Delta \mathrm{E}_{\mathrm{pac}}$ between the two anodic and cathodic peaks of $\mathrm{Fe}(\mathrm{CN})_{6}{ }^{-3 / 4-} \Delta \mathrm{E}_{\mathrm{pac}}(\mathrm{CPE})=340 \mathrm{mV}$ and $\Delta \mathrm{E}_{\mathrm{pac}}(\mathrm{Ag}-\mathrm{NPs} @$ $\mathrm{CPE})=20 \mathrm{mV}$, has likewise decreased significantly. In terms of the electron cinetics of this electrode and its interface this indicates that the electron transfer cinetics of the electrolyte/ electrode interface utilizing Ag-NPs@CPE is particularly rapid compared to the unmodified electrode (CPE).

In conclusion, the deposits obtained for electrolysis durations of $300 \mathrm{~s}$ allow to bring to the surface an electrocatalytic effect resulting in a faster charge transfer than graphite carbon alone, accompanied by an increase in the active surface, taking into account the results of the characterization by the $\mathrm{Fe}(\mathrm{CN})_{6}{ }^{-3 / 4-}$ redox probes. Scanning electron microscopy studies, on the other hand, reveal more homogenous and denser deposits after $300 \mathrm{~s}$ of electrolysis. This period of electrolysis was, therefore, preserved for silver deposits on carbon graphite paste, resulting in a greater quantitative efficiency of the electrolytic deposition of AgNPs.

\section{Application to the electrochemical detection of Nitrofurazone}

\subsection{Effect of scan rate}

At varied scan rates ranging from $20 \mathrm{mV} / \mathrm{s}$ to $200 \mathrm{mV} / \mathrm{s}$, cyclic voltammograms were obtained by a $10^{-3} \mathrm{M}$ reduction process of antimicrobial Nitrofurazone on the surface of an Ag-NPs@CPE electrode (Fig. 7A). The reduction peak of antimicrobial Nitrofurazone was influenced as the scan rate increased, with the current intensity of the peak rising and moving to higher cathodic potential values.

With a regressive equation $I p c(N F Z)=-3.9718 \mathrm{v}$ -123.59 and a correlation coefficient $R^{2}=0.988$, Fig. 6B. Demonstrates that the scan rate (v) was proportional to the change in the electrocatalytic current intensity of the nitrofurazone reduction peak. Figure $7 \mathrm{C}$ shows that the variation in current intensity of the Nitrofurazone peak as a function of the square root of the scanning speed $(\mathrm{v})^{1 / 2}$ is linear with a regressive equation $\mathrm{Ipc}(\mathrm{NFZ})=-108.94 \mathrm{v}^{1 / 2}-134.81$ and a correlation coefficient $\mathrm{R}^{2}=0.9936$. These results indicate that diffusion phenomena within the cellule of measurement control the evolution of the active surface of the Ag-NPs@ $\mathrm{CPE}$ electrode during the reduction reaction of antimicrobienne Nitrofurazone molecules. This phenomenon resembles what Yung-Shun Lu and All [23] observed in their study. It represents the best-case scenario for quantitative detections of the antimicrobial Nitrofurazone in real samples, as well as the best-case scenario for building a fast and efficient detection system for these molecules.

Figure 7 Represents the variation of the cathodic peak potential of the antibiotic reduction of Nitrofurazone as a function of the logarithm of the scan rate ( $\log v)$. The peak potentials vary linearly with the logarithm of the scan rate $\log (\mathrm{v})$, as shown in Fig. 6D. With a regression equation $\mathrm{Epc}(\mathrm{NFZ})=-0.02264 \mathrm{Log} \mathrm{v} / \mathrm{mV} \cdot \mathrm{s}^{-1}-0.2534$ and a correlation coefficient $\left(\mathrm{R}^{2}=0.988\right)$. This reveals that the peak potential of Nitrofurazone was very sensitive to the scan rate of electron transfer. Laviron demonstrated that the equation between Epc and Log (v) in the case of an irreversible system [24]

$E p c(N F Z)=E 0-\frac{R T}{\alpha n F}[0.780+\log (\sqrt{ } D / k)+\log (n F \alpha v / R T) 1 / 2$

$E p c(N F Z)=E p c+\frac{R T}{1-\alpha} n F \log (v)$

With $\mathrm{R}$ is the constant of perfect gas (9.31SI), $\mathrm{T}$ is the ambient temperature $(298 \mathrm{k})$, the constant of fardaye (96546C), $\alpha$ is the coefficient of electron transfer, $n$ is the number of electrons captured by the molecules of Nitrofurazone for the reduction on the electrocatalytic surface of our constructed sensor. 


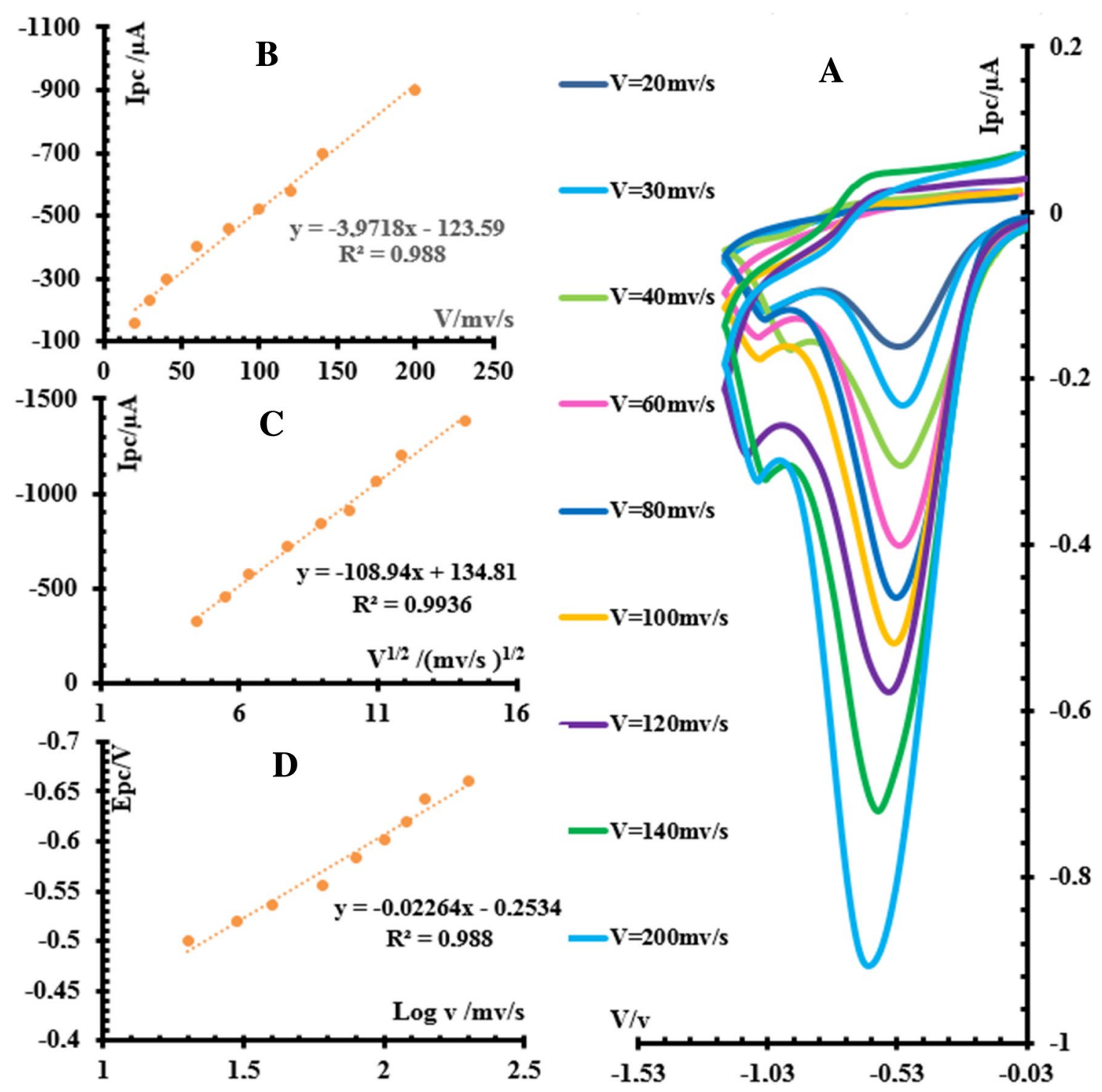

Fig. $7 \mathrm{CVs}$ response of $1 \mathrm{mM}$ the nitrofurazone to the Ag-NPs@ CPE constructed electrode with different scan rates $(20-100 \mathrm{mV} / \mathrm{s})$ at $\mathrm{pH}=7$ (A). The $\mathrm{I}_{\mathrm{PC}}$ calibration curve as a function of $\mathrm{v}(\mathrm{mV} / \mathrm{s})$. (B)

and plot of the Ipc as a function of $\mathrm{v}^{1 / 2} /(\mathrm{mV} / \mathrm{s})^{1 / 2}(\mathbf{C})$ and the Epc variation as a function of Log v. $\mathrm{mV} / \mathrm{s}(\mathbf{D})$

Given this, nitrofurazone antibacterial electrochemical action is totally irreversible, indicating an electron transfer coefficient of 0.61 . We calculate the number of electrons (n) transmitted in the electroreduction of the side chain attraction group of the nitrofurazone antimicrobial molecules by following the example of Bard and Faulkner from the pent achieved between $\operatorname{Epc}(\mathrm{NFZ})-\log (\mathrm{v} / \mathrm{mV} / \mathrm{s})$ and Lavirons, Eq. 2. The observations of A. Rahi et al. [7]. Are comparable with our conclusions.

As a result, during the catalytic reaction taking place in the area of the modified electrode and within the response time of the electrode, the number of electrons (n) transferred to the surface of the modified electrode for the electroreduction of the electroactive $\mathrm{NO}_{2}$ moiety of Nitrofurazone is equal to 3.88 (approximately equal to 4) under the best and most favorable operating conditions.

\subsection{Effect of $\mathrm{pH}$}

In the $\mathrm{pH}$ range of 4 to 10 we also examined at how the $\mathrm{pH}$ of the electrolyte solution affected the reduction current peak and cathodic potential peak of Nitrofurazone cyclic voltammograms were obtained at a $20 \mathrm{mv} \mathrm{s}^{-1}$ scan rate. The tompon phosphate solution at $\mathrm{pH}=7$ had the highest electrocatalytic reduction current of Nitrofurazone. A stabilization of the current intensity is evident in the cyclic voltammograms obtained in Fig. 8. Suggesting that the reduction process of Nitrofurazone is less kinetically favourable at higher $\mathrm{pH}$. At 

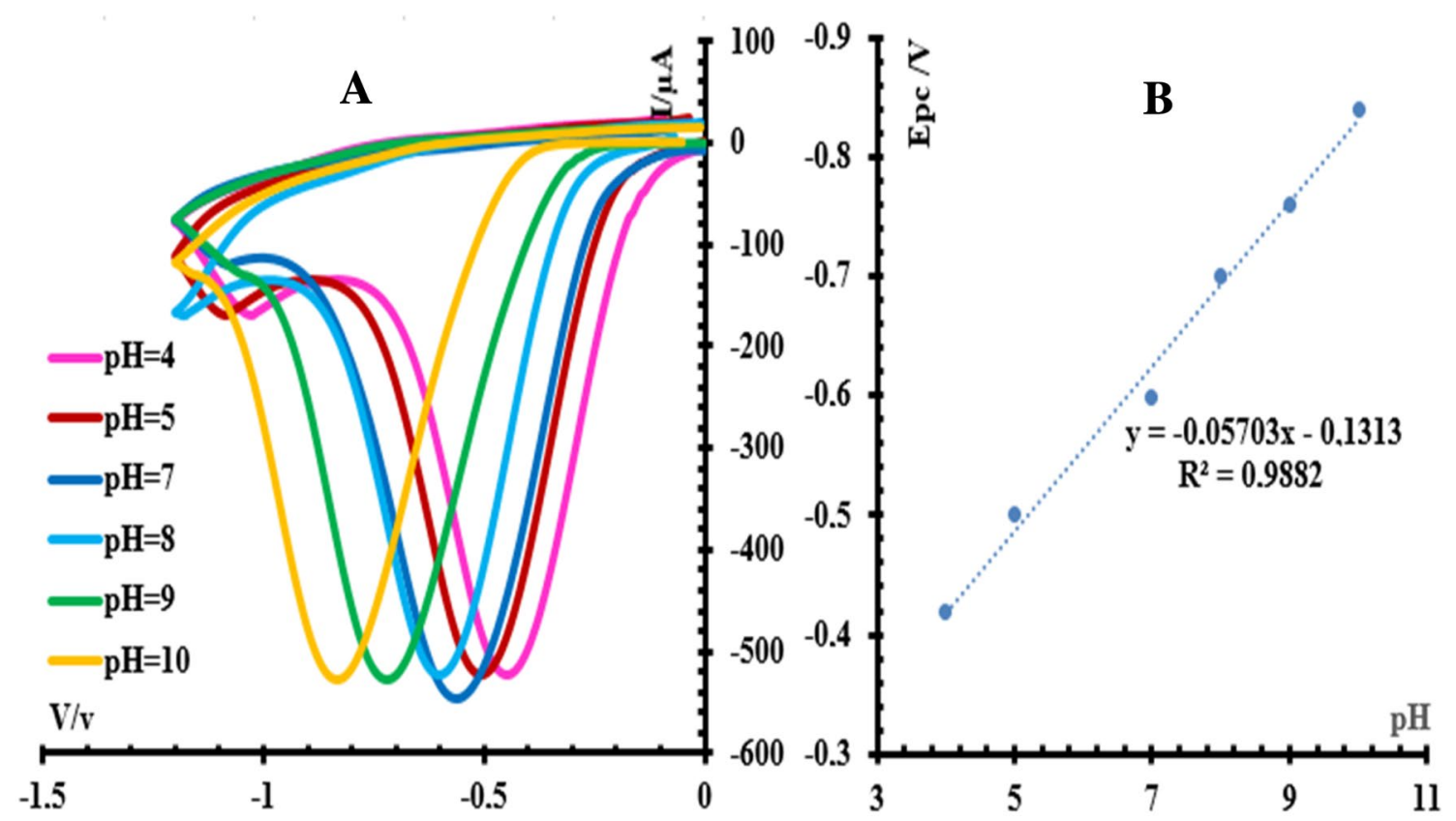

Fig. 8 A cyclic voltammograms (at $20 \mathrm{mV} / \mathrm{s}$ ) of $1.0 \times 10^{-3} \mathrm{M}$ Nitrofurazone in Ag-NPs@CPE constructed at different values of (pH=4 to 10). Inset: (B) Plot of $\mathrm{E}_{\mathrm{pc}}$ vs $\mathrm{pH}$

$\mathrm{pH}=7$ the maximum current peak was observed. Therefore, in the following tests $0.1 \mathrm{~mol} \mathrm{~L}^{-1}$ the tompon phosphate solution $(\mathrm{pH}=7)$ was used as the supporting electrolyte at the conditions initially described in this study.

Figure 8A Illustration It demonstrates the linearity observed between $\mathrm{pH}$ changes and the peak of Nitrofurazone reduction potential. Epc $(\mathrm{NTZ})=-0.05703 \mathrm{pH}-0.1313$ $\left(\mathrm{R}^{2}=0.9882\right)$ is the regression equation obtained showing that the slope obtained is almost comparable to the slope of the theoretical equation of Nernst. This result indicates that the number of protons participating in the reduction process of Nitrofurazone molecules on the electrode surface created by electrodeposition of $\mathrm{Ag}$ on graphite carbon paste is equal to the number of electrons $m / n=1$. The numerical value of the slope $(-0.057)$ obtained by applying the equation and the numerical value of the slope of the theoretical Nernst equation (Eq. 3) which are extremely near to each other are numerically justified :
${ }_{p c}(N T Z)=E^{\circ}-\left(\frac{2,303 m R T}{n F}\right) p H=E^{\circ}-0,059 \frac{m}{n} p H$

where $m$ is the number of proton, $n$ is the number of electrons.

When the reduction reaction is performed on the surface of the constructed electrode the electroactive $-\mathrm{NO}_{2}$ groups of the Nitrofurazone chain length are converted to $-\mathrm{NHOH}$.

Scheme 1: Shows the mechanism that adequately describes the Nitrofurazone electroreduction process on the electrocatalytic surface of the constructed Ag-NPs@ CPE sensor. This mechanism is very similar to the conclusions of the work of Yung-Shun Lu and All [23].
Scheme 1 The mechanism for the electroterduction reaction of Nitrofurazone on Ag-NPs@ CPE justification

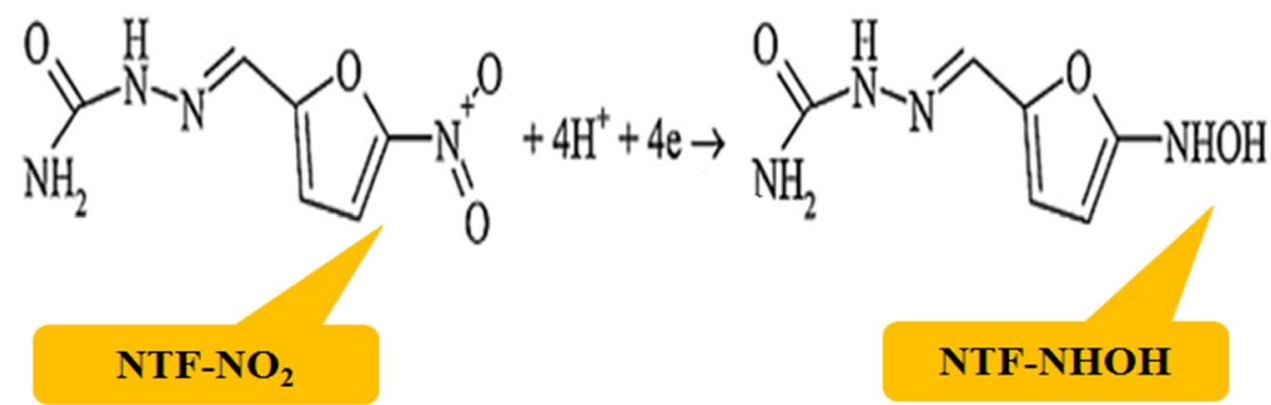




\subsection{Calibration curve}

The differential voltammetry technique was used to determine Nitrofurazone in the concentration range of $10^{-3} \mathrm{M}$ to $0.410^{-7} \mathrm{M}$ using the electrode constructed by electrodeposition under optimized experimental conditions (scan rate $20 \mathrm{mV} / \mathrm{s}, \mathrm{pH}=7$ applied potential $00 \mathrm{mV}$ to $-1200 \mathrm{mV}$ ) Fig. 9A. Illustrates the responses.

The calibration line obtained for the detection of Nitrofurazone is presented in Fig. 8B it expresses the intensity of the reduction current of Nitrofurazone molecules at the surface of our constructed sensor as a function of its concentration in the phosphate buffer medium.

As shown in the calibration line the intensity of the reduction current increases linearly with the increase of their concentration according to the following linear equation.

$\mathrm{Ipc}(\mathrm{NTZ})=-6.2766[\mathrm{NTZ}]-82.456$ and a regression coefficient $\left(\mathrm{R}^{2}=0.9954\right)$.

The limits of detection and quantification were calculated using the following equation: $\mathrm{LOD}=3 \sigma / \mathrm{m}$ and $\mathrm{LOQ}=10 \sigma / \mathrm{m}$ with $\sigma$ the standard deviation of the response according to IUPAC recommendations this value is the average of the deviations determined for 10 blank voltammograms, and $\mathrm{m}$ is the slope of the calibration curve. Application of the above relationship gives a detection and quantification limit of $10^{-8} \mathrm{M}$ and $10^{-7} \mathrm{M}$, respectively, for Nitrofurazone.

The performance of our Ag-NPs@CPE constructed electrode was compared with some modified electrodes cited in the literature for Nitrofurazone detection under similar conditions. We have summarized the comparison in Table 2.

\subsection{Effect of interferents}

The presence of non-target ions in real analytical samples may create problems with Nitrofurazone detection. As a result, we investigated the influence of various ions on the determination of $10^{-4} \mathrm{M}$ Nitrofurazone to evaluate the selectivity of a sensor constructed for electroanalysis of Nitrofurazone in a complex matrix and to identify potential issues owing to the presence of interfering ions. Table 3 shows the intensity of Nitrofurazone reduction current in the presence of interfering ions at 5 times the concentration of Nitrofurazone.

As a result of these observations, the presence of various interfering ions had little effect on the nitrofurazone measurement; the rate of signal change was in the range of 2-3\%. The Ag-NPs@CPE sensor exhibited high selectivity, indicating that it was well elaborated. These results were
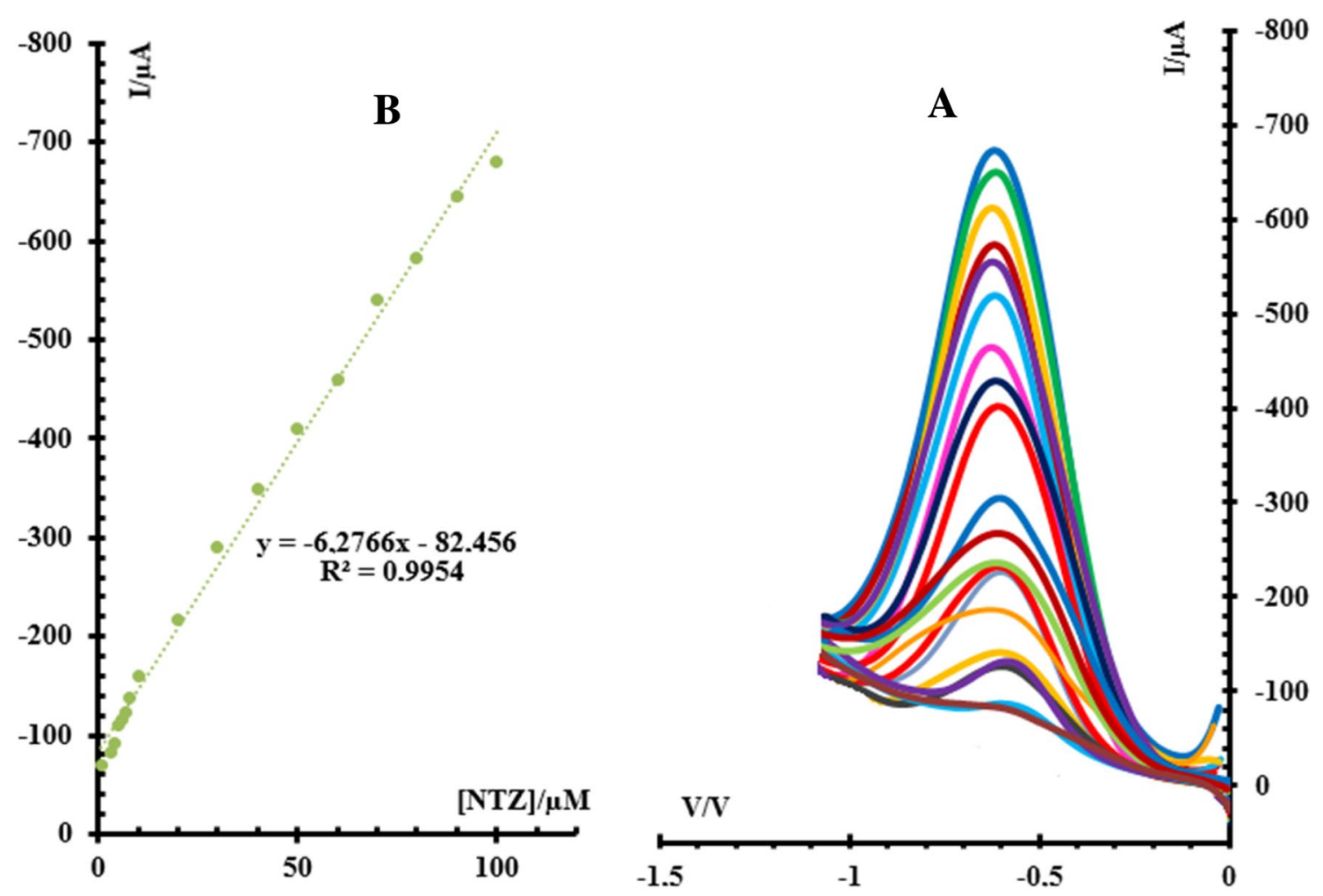

Fig. 9 Differential pulse voltammetry of Nitrofurazone in $0.1 \mathrm{M}$ the phosphate buffer $(\mathrm{pH}=7)$ for $\{\mathbf{1 0 0 ; 8 0 ; 7 0 ; 6 0 ; 5 0 ; ; 4 0 ; 3 0 ; 2 0 ; 1 5 ; 1 0 ; ~}$ $\mathbf{8 ; 6 ; 5 ; ; 4 ; 1 ; 0 . 6 ; 0 . 5 ; 0 . 0 4 ~} \boldsymbol{\mu M}\}$; (A), Inset: plots of electrocatalytic peak current as a function of Nitrofurazone concentration $(\mathrm{B})$ 
Table 2 Performance comparison of our constructed Ag-NPs@CPE electrode with different developed modified electrodes and techniques in the literature

\begin{tabular}{lllll}
\hline Electrod & Technique & Linear range $\mu \mathrm{M}$ & LOD $\mu \mathrm{M}$ & Ref \\
\hline Au-AuNR & DPV & $\mathbf{3 . 0 - 5 0 0}$ & $\mathbf{0 . 1 8}$ & {$[7]$} \\
PACBK/GCE & DPV & $\mathbf{1 5 - 8 0}$ & $\mathbf{0 . 0 2 8}$ & {$[25]$} \\
DUT-67/T-PPY-2/GCE & DPV & $\mathbf{9 . 0 8 - 3 5 4 . 0 8 ; 3 5 4 . 0 8 - 1 0 0 4 . 0 4}$ & $\mathbf{8 . 7}$ & {$[26]$} \\
Ag-SDS & Amp & $\mathbf{0 . 6 6 - 4 7 . 6 2 ; 4 7 . 6 2 - 9 3 0}$ & $\mathbf{0 . 3 7}$ & {$[23]$} \\
AuNPs/Gr/TFGE & DPV & $\mathbf{0 . 0 2 - 2 0 0 0}$ & $\mathbf{0 . 1 3}$ & {$[6]$} \\
HBDDE/GCE & DPV & $\mathbf{0 . 9 9 - 1 1}$ & $\mathbf{3 4 0}$ & {$[27]$} \\
Ag-NPS@CPE & DPV & $\mathbf{0 . 0 4 - 1 0 0 0}$ & $\mathbf{0 . 0 1 2}$ & This work
\end{tabular}

Table 3 Effect of coexisting substances on the detection of antimicrobial Nitrofurazone at $1.010^{-4} \mathrm{M}(n=3)$

\begin{tabular}{|c|c|c|c|c|c|c|c|}
\hline $\begin{array}{l}\text { Coexisting } \\
\text { substance }\end{array}$ & $\begin{array}{l}\text { Concentration de } \\
\text { Coexisting substance } \\
\left(\mathrm{mmol} \mathrm{L}^{-1}\right)\end{array}$ & $\begin{array}{l}\text { Change of } \\
\text { peak current } \\
(\%)\end{array}$ & $\begin{array}{l}\text { Changement du } \\
\text { potentiel de pic } \\
(\%)\end{array}$ & $\begin{array}{l}\text { Coexisting } \\
\text { substance }\end{array}$ & $\begin{array}{l}\text { Concentration de } \\
\text { Coexisting substance } \\
\left(\mathrm{mmol} \mathrm{L}^{-1}\right)\end{array}$ & $\begin{array}{l}\text { Changement du } \\
\text { potentiel de pic } \\
(\%)\end{array}$ & $\begin{array}{l}\text { Change of } \\
\text { peak current } \\
(\%)\end{array}$ \\
\hline $\mathrm{Na}^{+}$ & 1 & -0.94 & +0.32 & $\mathrm{Cl}^{-}$ & 1 & -0.5 & 0.34 \\
\hline $\mathrm{Al}^{2+}$ & 1 & -1.40 & +0.11 & $\mathrm{SO}_{4}{ }^{2-}$ & 1 & -0.3 & -0.4 \\
\hline $\mathrm{Ni}^{2+}$ & 1 & -1.65 & +0.15 & $\mathrm{NO}_{3}^{-}$ & 1 & +0.44 & -1.03 \\
\hline $\mathrm{Fe}^{3+}$ & 1 & -1.6 & +0.22 & $\mathbf{Z n}^{3+}$ & 1 & +0.65 & -1.76 \\
\hline $\mathrm{Cu}^{2+}$ & 1 & -1.2 & +0.26 & $\mathrm{Mg}^{2+}$ & 1 & +0.71 & -0.4 \\
\hline $\mathrm{Ca}^{2+}$ & 1 & -0.57 & +0.26 & $\mathrm{CO}_{3}{ }^{2-}$ & 1 & +0.65 & -1.41 \\
\hline $\mathrm{K}^{+}$ & 1 & 1.88 & -0.34 & $\mathrm{Mn}^{2+}$ & $\mathbf{1}$ & +2.81 & -1.25 \\
\hline
\end{tabular}

obtained under experimental conditions that were optimized from the beginning of the work performed.

We can also see that the rate of recovery is more than $90 \%$. This is extremely good in terms of the methods effectiveness and the analytical performance of the constructed electrode utilized for the reduction of Nitrofurazone detection. According to these tests the presence of different interfering ions has little effect on the detection of Nitrofurazone suggesting that the Ag-NPs@CPE sensor has good selectivity and that these sensors are thus useful in the analysis of Nitrofurazone in complex matrices such as human urine or drinking water under rigorous operating conditions.

\subsection{Repeatability and reproducibility of Ag-NPs@ CPE}

By analyzing $10^{-4} \mathrm{M}$ of Nitrofurazone prepared in phosphate buffer solution $(\mathrm{pH}=7)$, the repeatability of measurements for Nitrofurazone detection using the modified Ag-NPs@ CPE electrode manufactured under optimal experimental conditions was investigated. The relative standard deviation (RSD\%) estimated after ten consecutive measurements with the same electrode was $3.2 \%$ on average and the result of these measurements was acceptable and sufficient for the detection of Nitrofurazone.

A series of eight electrodes were prepared to verify the repeatability of its Ag-NPs@CPE electrode, which was constructed using comparable techniques. These were utilized to detect Nitrofurazone at a concentration of $10^{-4} \mathrm{M}$. The relative standard deviation of the eight electrode observations is calculated to be $3.6 \%$.

These results clearly indicate that the Ag-NPs@CPE electrode has excellent repeatability and reproducibility.

\section{Analytical application}

The built sensor was tested for detecting Nitrofurazone in real-world contaminated samples. We examined two different types of samples: urine and tap water. We examined the samples with the suggested sensor before they were contaminated, and the findings revealed that the infected samples had no reduction peaks corresponding to the electroactive group $\mathrm{NO}_{2}$ of Nitrofurazone. These experiments were carried out in our research center.

A volume of $40 \mathrm{~mL}$ of the solution obtained from real samples contaminated with the antibiotic Nitrofurazone was poured into the electrochemical cell and analyzed by the sensor built under previously optimized experimental conditions. The percentage of Nitrofurazone spiked in the analyzed actual samples that were tested was calculated using the standard addition method.

Table 4 shows the results obtained for the various samples. The recoveries of Nitrofurazone found in various contaminated samples range from 97 to $102 \%$, with a relative standard deviation (R.S.D.) $(n=3)$ of less than $4 \%$, 
Table 4 Results of antimicrobial detection of Nitrofurazone in selected real samples $(n=3)$ that were analyzed in a systematic manner

\begin{tabular}{lllll}
\hline Sample & Spiked $(\mu \mathrm{M})$ & Found $(\mu \mathrm{M})$ & Accuracy $\%$ & R.S.D $\%(n=3)$ \\
\hline Urine + NTZ & 10 & 9.887 & 99.21 & 1.33 \\
& 5 & 4.875 & 98.02 & 3.44 \\
Tap water + NTZ & 1 & 0.962 & 97.8 & 1.24 \\
& $\mathbf{2}$ & 4.977 & 98.7 & 1.25 \\
& 1 & 2.53 & 102.06 & 1.43 \\
& 1.09 & 101.02 & 1.11 \\
\hline
\end{tabular}

demonstrating that the constructed Ag-NPs@CPE sensor has excellent practical cost effectiveness for the electroanalysis of antimicrobial Nitrofurazone in real samples under real working conditions.

\section{Conclusions}

In this work, we used electrodeposition to efficiently develop an Ag-NPs@CPE electrochemical sensor to detect the antimicrobial Nitrofurazone in real samples. Due to its simplicity and fast implementation. The electrochemical fabrication strategy offers several advantages to facilitate the direct electrodeposition of silver on the working electrode. We have also studied and optimized all the parameters allowing the formation of a silver film on the carbon graphite. The silver film constructed on the carbon paste was revealed to have high electrical conductivity, and strong electrocatalytic property, The electrochemical behavior of nitrofurazone is completely irreversible with a coherently elaborated sensor-level diffusion controlled surface transfer mechanism. The number of electrons and protons being equal during the reaction of nitrofurazone effected on the surface of our sensor. The Ag-NPs@CPE analytical performance demonstrates excellent repeatability and selectivity. The differential pulse voltammetry technique revealed that the Ag-NPs@CPE has a very wide detection range for Nitrofurazone, ranging from $10^{-3} \mathrm{M}$ to $0.410^{-7} \mathrm{M}$ with a very low detection limit of $1.2 \times 10^{-8} \mathrm{M}$. The constructed sensor was tested for the detection of nitrofurazone in real human urine and tap water samples and its performance was satisfactory in terms of detection efficiency.

Acknowledgements The authors wish to express their gratitude to the University ibn zohr of Agadir. This research was carried out thanks to their material support from the university.

\section{Declarations}

Conflict of interest The authors declare that they have no conflict of interest.

\section{References}

1. Hou S-L, Dong J, Jiang X-L, Jiao Z-H, Wang C-M, Zhao B (2018) Interpenetration-dependent luminescent probe in indium-organic frameworks for selectively detecting nitrofurazone in water. Anal Chem 90:1516-1519. https://doi.org/10. 1021/acs.analchem.7b05088

2. Khong S-P, Gremaud E, Richoz J, Delatour T, Guy PA, Stadler RH, Mottier P (2004) Analysis of matrix-bound nitrofuran residues in worldwide-originated honeys by isotope dilution high-performance liquid chromatography-tandem mass spectrometry. J Agric Food Chem 52:5309-5315. https://doi.org/10. 1021/jf0401118

3. Cheng J, Li Y, Zhong J, Lu Z, Wang G, Sun M, Jiang Y, Zou P, Wang X, Zhao Q, Wang Y, Rao H (2020) Molecularly imprinted electrochemical sensor based on biomass carbon decorated with MOF-derived $\mathrm{Cr} 2 \mathrm{O} 3$ and silver nanoparticles for selective and sensitive detection of nitrofurazone. Chem Eng J 398:125664. https://doi.org/10.1016/j.cej.2020.125664

4. Ying-Ying W, Zhen-Hu X (2016) Multi-walled carbon nanotubes and powder-activated carbon adsorbents for the removal of nitrofurazone from aqueous solution. J Dispersion Sci Technol 37:613-624. https://doi.org/10.1080/01932691.2014. 981337

5. Gupta A, Upadhyay NK, Parthasarathy S, Rajagopal C, Roy PK (2013) Nitrofurazone-loaded PVA-PEG semi-IPN for application as hydrogel dressing for normal and burn wounds. J Appl Polym Sci 128:4031-4039. https://doi.org/10.1002/app.38594

6. He B, Liu H (2019) Electrochemical determination of nitrofuran residues at gold nanoparticles/graphene modified thin film gold electrode. Microchem J 150:104108. https://doi.org/10.1016/j. microc. 2019.104108

7. A. Rahi, N. Sattarahmady, R. Dehdari Vais, H. Heli, Sonoelectrodeposition of gold nanorods at a gold surface - Application for electrocatalytic reduction and determination of nitrofurazone, Sensors and Actuators B: Chemical. 210 (2015) 96-102. https://doi.org/10.1016/j.snb.2014.12.090.

8. Kokulnathan T, Wang T-J (2019) Synthesis and characterization of 3D flower-like nickel oxide entrapped on boron doped carbon nitride nanocomposite: An efficient catalyst for the electrochemical detection of nitrofurantoin. Compos B Eng 174:106914. https://doi.org/10.1016/j.compositesb.2019.106914

9. Horie M, Tabara Y, Ishii R, Hashimoto K, Yamagishi Y (2008) Determination of nitrofurazone in livestock products and seafoods by liquid chromatography-tandem mass spectrometry. J Food Hyg Soc Jpn 49:204-210. https://doi.org/10.3358/shokueishi.49.204

10. Wang B, Liu J-H, Yu J, Lv J, Dong C, Li J-R (2020) Broad spectrum detection of veterinary drugs with a highly stable metal-organic framework. J Hazard Mater 382:121018. https:// doi.org/10.1016/j.jhazmat.2019.121018

11. C.-W. Tsai, C.-H. Tang, W.-H. Wang, Quantitative determination of four nitrofurans and corresponding metabolites in the fish muscle by liquid chromatography-electrospray 
ionization-tandem mass spectrometry, Journal of Food and Drug Analysis. 18 (2020). https://doi.org/10.38212/2224-6614.2280.

12. I. Svancara, K. Kalcher, A. Walcarius, K. Vytras, Electroanalysis with Carbon Paste Electrodes, 0 ed., CRC Press, 2012. https://doi.org/10.1201/b11478.

13. S.J. Malode, K.P. K., N.P. Shetti, K.R. Reddy, Highly sensitive electrochemical assay for selective detection of Aminotriazole based on TiO2/poly(CTAB) modified sensor, Environmental Technology \& Innovation. 21 (2021) 101222. https://doi.org/10. 1016/j.eti.2020.101222.

14. Shetti NP, Malode SJ, Vernekar PR, Nayak DS, Shetty NS, Reddy KR, Shukla SS, Aminabhavi TM (2019) Electro-sensing base for herbicide aclonifen at graphitic carbon nitride modified carbon electrode - Water and soil sample analysis. Microchem J 149:103976. https://doi.org/10.1016/j.microc.2019.103976

15. Shanbhag MM, Shetti NP, Kulkarni RM, Chandra P (2020) Nanostructured $\mathrm{Ba} / \mathrm{ZnO}$ modified electrode as a sensor material for detection of organosulfur thiosalicylic acid. Microchem J 159:105409. https://doi.org/10.1016/j.microc.2020.105409

16. N.P. Shetti, S.J. Malode, D. Ilager, K. Raghava Reddy, S.S. Shukla, T.M. Aminabhavi, A Novel Electrochemical Sensor for Detection of Molinate Using ZnO Nanoparticles Loaded Carbon Electrode, Electroanalysis. 31 (2019) 1040-1049. https://doi.org/ 10.1002/elan.201800775.

17. Bukkitgar SD, Shetti NP (2017) Fabrication of a $\mathrm{TiO}_{2}$ and clay nanoparticle composite electrode as a sensor. Anal Methods 9:4387-4393. https://doi.org/10.1039/C7AY01068K

18. Pavamana M, Shetti NP, Malode SJ, Bukkitgar SD (2019) Nano level detection and analysis of an antiviral drug at $\mathrm{ZnO}$ nanoparticles modified sensor. Materials Today: Proceedings 18:15681573. https://doi.org/10.1016/j.matpr.2019.07.086

19. Vernekar PR, Shetti NP, Shanbhag MM, Malode SJ, Malladi RS, Reddy KR (2020) Novel layered structured bentonite clay-based electrodes for electrochemical sensor applications. Microchem J 159:105441. https://doi.org/10.1016/j.microc.2020.105441

20. D.R. Kulkarni, S.J. Malode, K. Keerthi Prabhu, N.H. Ayachit, R.M. Kulkarni, N.P. Shetti, Development of a novel nanosensor using Ca-doped $\mathrm{ZnO}$ for antihistamine drug, Materials Chemistry and Physics. 246 (2020) 122791. https://doi.org/10.1016/j.match emphys.2020.122791.
21. Behera S, Behura R, Mohanty M, Dinda R, Mohanty P, Verma AK, Sahoo SK, Jali BR (2020) Spectroscopic, cytotoxicity and molecular docking studies on the interaction between 2,4-dinitrophenylhydrazine derived Schiff bases with bovine serum albumin. Sensors International 1:100048. https://doi.org/10.1016/j.sintl. 2020.100048

22. Zoubir J, Bakas I, Assabbane A (2021) A simple platform for the electro-catalytic detection of the dimetridazole using an electrochemical sensor fabricated by electro-deposition of $\mathrm{Ag}$ on carbon graphite: application: orange juice, tomato juice and tap water. Heliyon 7:e07542. https://doi.org/10.1016/j.heliyon.2021.e07542

23. Lu Y-S, Pan W-Y, Hung T-C, Hsieh Y-T (2020) Electrodeposition of silver in a ternary deep eutectic solvent and the electrochemical sensing ability of the Ag-modified electrode for nitrofurazone. Langmuir 36:11358-11365. https://doi.org/10.1021/acs.langmuir. 0c02213

24. Jain R, Gupta VK, Jadon N, Radhapyari K (2010) Voltammetric determination of cefixime in pharmaceuticals and biological fluids. Anal Biochem 407:79-88. https://doi.org/10.1016/j.ab.2010. 07.027

25. Chen C, Chen W, Jiang J, Qian L, Qiu F (2021) Preparation and application of nitrofuran sensor in aquaculture water. Int $\mathrm{J}$ Environ Anal Chem 101:1099-1115. https://doi.org/10.1080/03067319. 2019.1676423

26. Wang H, Bo X, Zhou M, Guo L (2020) DUT-67 and tubular polypyrrole formed a cross-linked network for electrochemical detection of nitrofurazone and ornidazole. Anal Chim Acta 1109:1-8. https://doi.org/10.1016/j.aca.2020.03.002

27. M.S. da S. Julião, E.I. Ferreira, N.G. Ferreira, S.H.P. Serrano, Voltammetric detection of the interactions between RNO2- and electron acceptors in aqueous medium at highly boron doped diamond electrode (HBDDE), Electrochimica Acta. 51 (2006) 5080-5086. https://doi.org/10.1016/j.electacta.2006.03.054.

Publisher's Note Springer Nature remains neutral with regard to jurisdictional claims in published maps and institutional affiliations. 